\title{
Revision of Gorlin constant for calculation of mitral valve area from left heart pressures ${ }^{1}$
}

\author{
K. E. Hammermeister, J. A. Murray, and J. R. Blackmon \\ From the Cardiology Service, University Hospital, and the Cardiovascular Disease Service, \\ VA Hospital, Seattle, Washington, U.S.A.
}

The constant $(C=3 I)$ in the hydraulic formula for calculation of mitral valve area was originally empirically determined by comparison of measured valve area at operation or necropsy with right heart catheterization data. This constant corrects, among other things, for overestimation of the diastolic filling and mitral valve gradient derived from a peripheral arterial tracing and pulmonary capillary wedge pressure, respectively. With left heart catheterization these measurements can be made directly and more accurately. In this study through simultaneous measurement of pulmonary capillary wedge, peripheral arterial, left atrial, and left ventricular pressures, a new constant $(K=40)$ was derived which should provide a more accurate estimation of mitral valve orifice area when left heart data are used.

The hydraulic formula for the calculation of mitral valve area (MVA) developed by Gorlin and Gorlin (I95I) contains a constant (C) empirically calculated by comparison of mitral valve orifice area estimated at operation in II patients with cardiac output (CO), pulmonary capillary wedge pressure (PC), and diastolic filling period measured peripherally from the brachial artery (pDFP). Left ventricular diastolic pressure was assumed to average $5 \mathrm{mmHg}$, yielding a mitral valve gradient of PC-5 and the following formula:

$$
M V A=\frac{C O}{(\mathrm{pDFP})(\mathrm{HR})(\mathrm{C})(\sqrt{\overline{\mathrm{PC}}-5})}
$$

where $H R$ is heart rate. The constant, $C$, includes 5 factors: (I) conversion of pressure from $\mathrm{mmHg}$ to $\mathrm{cm} \mathrm{H}_{2} \mathrm{O}(\mathrm{I} \cdot \mathrm{I} 7)$, (2) deviation of the mitral valve from the 'perfect orifice' for which the formula is valid, (3) overestimation of the actual diastolic filling period (cDFP) because pDFP includes part of the isovolumic contraction and relaxation time, (4) overestimation of the actual mitral valve gradient by PC-5, and (5) $\sqrt{2 \mathrm{gg}}$ or 44.5 (g - gravity acceleration $\left.=980 \mathrm{~cm} / \mathrm{sec}^{2}\right)$. The constant, $\mathrm{C}$, derived from these data (the best available with catheterization techniques then in use) was calculated to be 31 , the number commonly used in this formula.

Received 3I July 1972.

1 These studies have been supported by grants from the National Heart Institute and Division of Chronic Diseases, Department of Health, Education and Welfare.
With the development of techniques for retrograde and transseptal catheterization of the left side of the heart, the pressure gradient across the mitral valve (LA-LV) and CDFP are commonly measured directly from left atrial (LA) and left ventricular (LV) pressure tracings. The constant, $\mathrm{C}$, originally estimated for use with indirect measurements will, when used with left heart data, result in an overestimation of mitral valve orifice area. It was the purpose of this study to arrive at a new value for this constant for use with left heart data. For clarity this new constant will be referred to as $K$, yielding the formula:

$$
\operatorname{MVA}=\frac{\mathrm{CO}}{(\mathrm{cDFP})(\mathrm{HR})(\mathrm{K})(\sqrt{\overline{\mathrm{LA}}-\mathrm{LV}})}
$$

\section{Methods}

One way to arrive at a value for $K$ would be to repeat the study of Gorlin and Gorlin (I95I) comparing mitral valve orifice area estimated at operation or measured at necropsy with calculated orifice area from left heart catheterization data obtained shortly before. However, due to the inaccuracies of measuring the mitral orifice at operation and the paucity of intact necropsy specimens, this method was deemed impractical. For the purposes of this study, it was assumed that the value for $C$ of $3 I$ for use with PC- 5 and pDFP was correct. Since, regardless of the technique of measurement, the mitral orifice is the same; then equation ( $I$ ) equals equation (2) in a given patient. Solving for $K$, the following formula is derived: 


$$
\mathrm{K}=\frac{(\mathrm{pDFP})(\sqrt{\mathrm{PC}-5})(3 \mathrm{I})}{(\mathrm{cDFP})(\sqrt{\overline{\mathrm{LA}}-\mathrm{LV}})}
$$

All four variables of equation (3) were measured from simultaneously recorded LA, LV, PC, and radial arterial pressures in 15 patients with predominant mitral stenosis undergoing diagnostic catheterization. Each patient, having given his informed consent, was studied fasting, supine, resting, and with mild sedation. One patient was studied also during isoprenaline infusion to make a total of 16 observations. Eight patients were in atrial fibrillation and 7 in sinus rhythm. A radial needle was introduced percutaneously. An 8F Cournand catheter was passed into the pulmonary artery via an antecubital vein. The left ventricle was catheterized retrogradely with an 8F Gensini catheter introduced percutaneously into the femoral artery. The left atrium was entered via transseptal puncture with an $8 \mathrm{~F}$ Brockenbrough catheter originally introduced percutaneously into the right femoral vein. All four pressures were measured simultaneously with four matched $\mathrm{P}_{23} \mathrm{Db}$ Statham pressure transducers and recorded optically on a Honeywell 1508 recorder. Mean PC was obtained electronically. Since catheter lengths were approximately the same, no attempt was made to correct the delay in transmission of pressure wave from cardiac chamber to transducer.

A total of 75 beats were each analysed separately. The true mean gradient across the mitral valve was obtained by planimetry from the superimposed LA and LV pressure curves. The cDFP was measured between the crossing of these two curves. The pDFP was measured from the dicrotic notch to the beginning of the upstroke of the next beat in the peripheral arterial pressure tracing. Those tracings in which the dicrotic notch was not clearly discernible were discarded. Fig. I is an example of a recording with four simultaneous pressures.
Mean, standard deviations, correlation coefficients, and regression equations were calculated with the aid of a computer. The significance of the correlation coefficient and the difference between the means of groups of paired variables were analysed using the Student $t$ test.

\section{Results}

The Table shows the mean and standard deviations of the corrected constant $(K)$ for use with left heart data and the variables from which it was derived. In Fig. $2, \mathrm{~K}$ is plotted against the heart rate of the individual cardiac cycles from which the calculation was made. $\mathrm{K}$ correlates significantly $(r=-0.544$, $\mathrm{P}<0.00 \mathrm{I}$ ) with heart rate, decreasing as heart rate

\section{TABLE Pressure gradients and diastolic filling} periods

\begin{tabular}{lc}
\hline Variable & Mean $\pm S D$ \\
\hline K & $39.9 \pm 7.7$ \\
PC-5 & $16.2 \pm 4.6 \mathrm{mmHg}$ \\
LA-LV & $13.2 \pm 5.2 \mathrm{mmHg}$ \\
$\Delta$ P & $3.0 \pm 4.2 \mathrm{mmHg}$ \\
pDFP & $0.42 \pm 0.16 \mathrm{sec}$ \\
cDFP & $0.38 \pm 0.05 \mathrm{sec}$ \\
$\Delta$ DFP & $0.04 \pm 0.03 \mathrm{sec}$
\end{tabular}

Abbreviations: SD - I standard deviation; $\mathrm{K}$ - corrected constant; $\Delta \mathbf{P}$ - difference between true mitral gradient (LA-LV) and that estimated by subtracting $5 \mathrm{mmHg}$ from pulmonary capillary pressure (PC-5); $\triangle \mathrm{DFP}$ - difference between true diastolic filling period (cDFP) and that estimated from peripheral arterial tracing (pDFP).

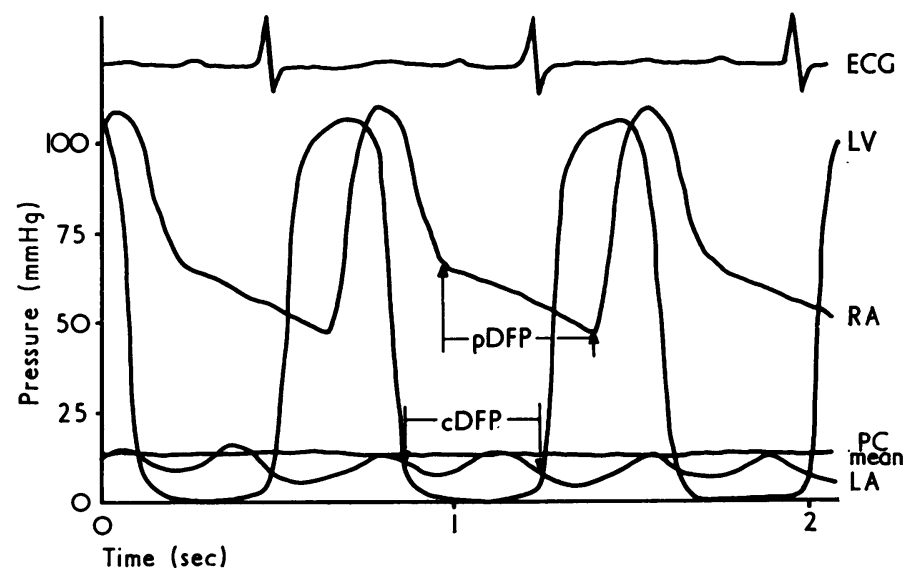

FI G. I Shows four simultaneously recorded pressures, radial artery $(R A)$, left ventricular $(L V)$, mean pulmonary capillary (PC mean), and left atrial $(L A)$ and the electrocardiogram (ECG) from which measurements of central diastolic filling period (cDFP) and peripheral diastolic filling period $(p D F P)$ were made. Gradients were determined from $L A, L V$, and $P C$ mean tracings as described in the text. 


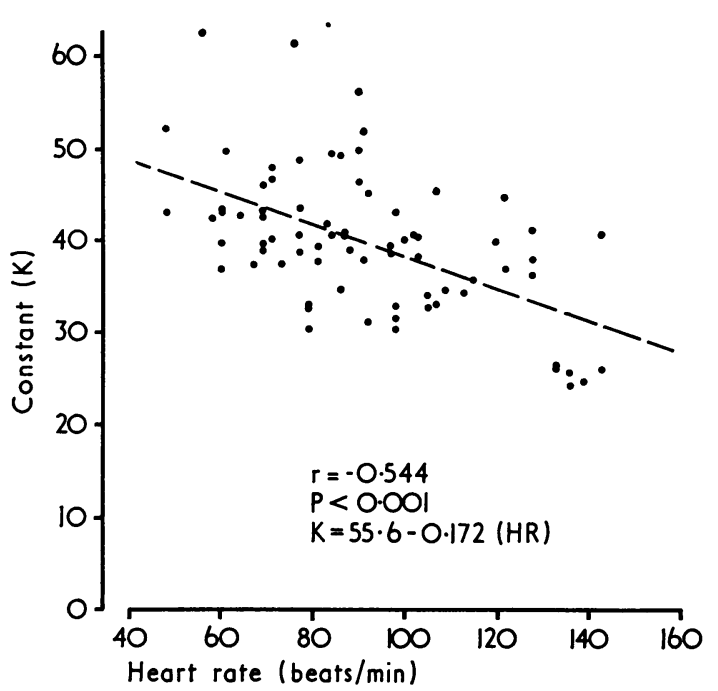

FIG. 2 Plot of the corrected constant $(K)$ against heart rate.

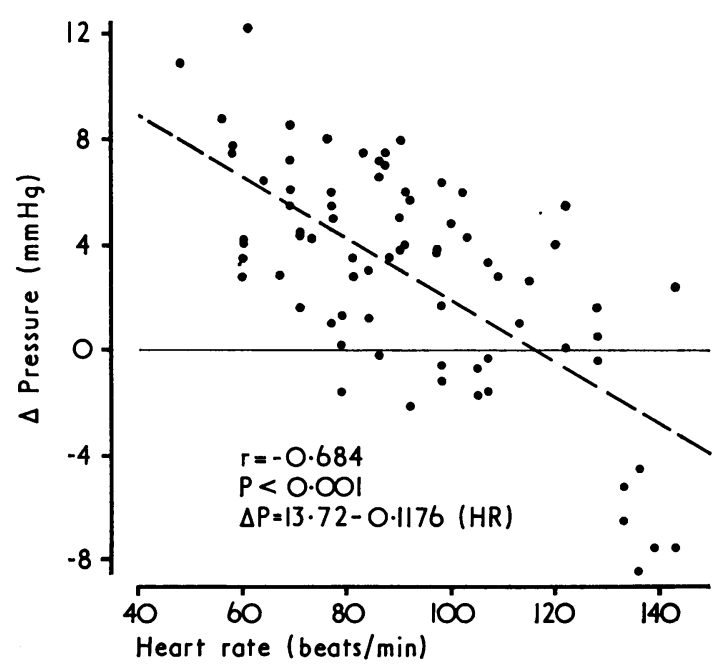

FIG. 3 Plot of the difference between PC-5 and $L A-L V(\Delta$ Pressure $)$ against heart rate.

increases. This is accounted for by the increasing difference between central and peripherally measured mitral valve gradient at slower heart rates (Fig. 3) and increasing difference between centrally and peripherally measured DFP with slower heart rate (Fig. 4).

There is no significant difference in $\mathrm{K}$ when calculated from patients in sinus rhythm $(41 \cdot 0)$ and from patients in atrial fibrillation (39.4).
Fig. 5 demonstrates a curvilinear relation between both central and peripheral measurements of diastolic filling period and heart rate. Fig. 6 shows a straight line relation between both pDFP and cDFP and the reciprocal of heart rate or the RR interval. These data could be used to estimate pDFP or cDFP if actual measurement were not possible, i.e. a peripheral arterial pressure tracing which does not show a dicrotic notch.

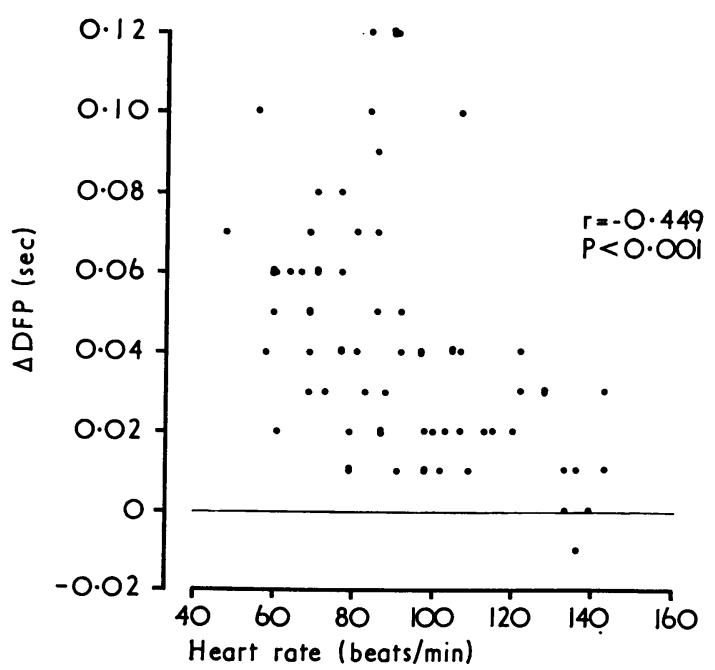

FIG. 4 Plot of the difference between peripherally measured diastolic filling period and centrally measured diastolic filling period $(\triangle D F P)$ against heart rate.

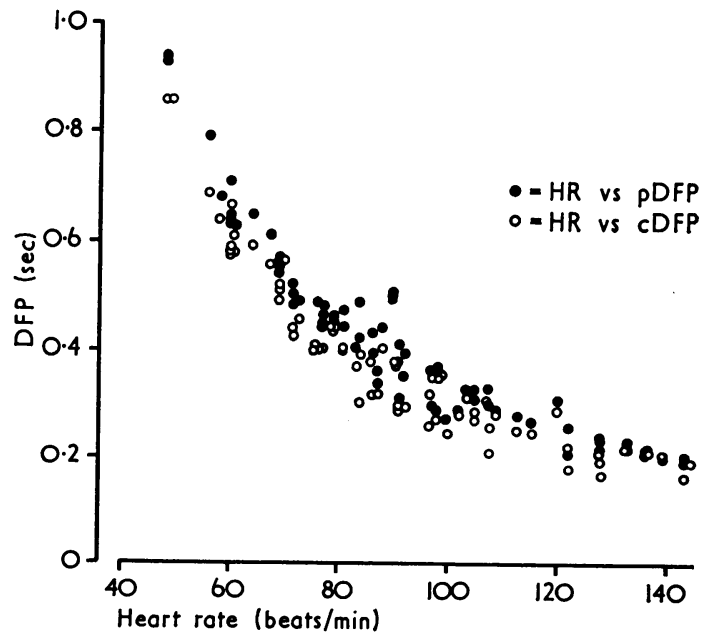

FIG. 5 Plot of peripheral diastolic filling period $(p D F P)$ in solid circles and central diastolic filling period (cDFP) in open circles against heart rate. 


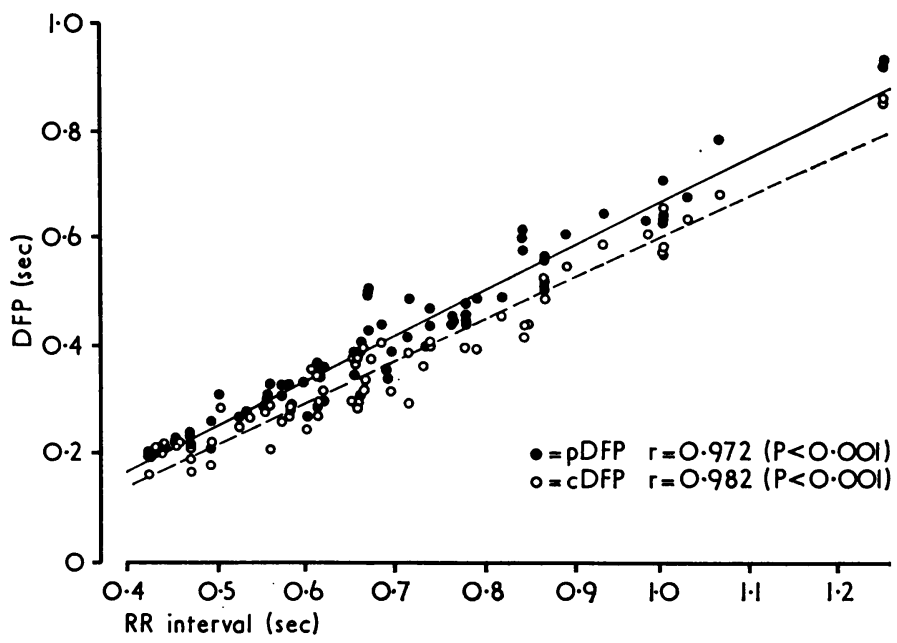

FIG. 6 Plot of peripheral diastolic filling period ( $p D F P$ ) in closed circles and the central diastolic filling period (cDFP) in open circles against the reciprocal of heart rate or $R R$ interval.

\section{Discussion}

Assuming the previous constant, $\mathrm{C}=3 \mathrm{r}$, to be valid for use with indirect measurements from right heart catheterization data, a new constant, $K=40$, has been derived for use with direct measurements from left heart catheterization data. The approximately 30 per cent increase in $\mathrm{K}$ over the previous $\mathrm{C}$ is accounted for by PC-5 overestimating the true mitral gradient (LA-LV) by an average of $3.0 \pm 4 \cdot 2$ mmHg $(\sqrt{\mathrm{PC}-5}$ overestimates $\sqrt{\mathrm{LA}-\mathrm{LV}}$ by 0.39 or II $\%$ ) and pDFP overestimating CDFP by $0.04 \mathrm{sec}$ (12\%). The differences between PC-5 and LA-LV and between pDFP and cDFP are statistically significant $(P<0.00 r)$.

Fox et al. (1956) measured LA, LV, aortic, and PC pressures in 5 patients with mitral stenosis and reported values for mean PC, mean diastolic LA, and mean diastolic LV pressures and diastolic filling periods measured centrally and from aortic pressure tracings. By making the appropriate calculations, we note that their data are compatible with ours in that PC-5 overestimates LA-LV (by $6.2 \mathrm{mmHg}$ ), and pDFP overestimates cDFP (by $0.08 \mathrm{sec}$ or $24 \%$ ). Substituting their recalculated data into our formula (3), we derive a value for $\mathrm{K}$ of 55 . In every case the directional changes are the same, but the magnitude of change is much greater than our data. This may be due to the fact that their pressures were not measured simultaneously despite the fact that 3 of the 5 patients were in atrial fibrillation. These authors note that using left heart data and a value for $\mathrm{C}$ of 3I, the calculated mitral valve area was consistently greater than the surgeon's estimate, an identical conclusion to ours.
While the estimated mitral gradient from right heart pressure exceeded the actual gradient by an average of $3 \mathrm{mmHg}$, the relation between mean PC and mean LA pressures is substantially closer. In 27 observations in 20 patients mean LA was 17.9 $\mathrm{mmHg}$, and mean PC was $19.0 \mathrm{mmHg}(r=0.95)$, the average difference being only $I \cdot I \mathrm{mmHg}$ $(P<0.05)$. Thus, measuring the mitral gradient from simultaneously recorded PC and LV pressures would overestimate the gradient only slightly, obviating the need for transseptal catheterization. The similarity between PC and LA pressures has been well documented previously (Connolly et al., 1953; Werkö et al., 1953; Dickens et al., 1957). If PC pressure is replotted to correct for delay in transmission through the lungs (approximately $0.08 \mathrm{sec}$ ), then DFP measured from superimposed PC and LV should be accurate. Thus the new constant, $K=40$, would be suitable for use with data obtained from simultaneously recorded $\mathrm{PC}$ and $\mathrm{LV}$ pressures.

The corrected constant, $\mathrm{K}$, appears to decrease with increasing heart rate (Fig. 2) because the error in estimation of gradient (Fig. 3) and diastolic filling period (Fig. 4) from right heart data decreases with increasing heart rate. However, this may be only apparent, as it may be that the original constant, C, should increase with increasing heart rate as the error for which it is to correct decreases. The relation of $\mathrm{C}$ to heart rate has not been studied. Thus, the authors recommend the use of a single constant, $\mathrm{K}=40$, with left heart data.

Left ventricular catheterization with or without left atrial catheterization permits a more accurate determination of the haemodynamics of mitral 
stenosis. If the gradient is small, assuming the mean $\mathrm{LV}$ diastolic pressure to be $5 \mathrm{mmHg}$ may intrcduce large errors. Therefore, direct left heart haemodynamic data is preferable for the assessment of mitral stenosis. The use of the original constant developed for use with right heart data will result in overestimation of the mitral valve area by $20-30$ per cent. The use of $\mathrm{K}=40$ with left heart data should give a more accurate estimate of mitral valve area.

The authors wish to acknowledge the technical assistance of Mr. Richard Brooks.

\section{References}

Connolly, D. C., Tompkins, R. G., Lev, R., Kirklin, J. W. and Wood, E. H. (1953). Pulmonary-artery wedge pressures in mitral valve disease; relationship to left atrial pressures. Proceedings of the Staff Meetings of the Mayo Clinic, 28, 72.
Dickens, J., Raber, G., Woldow, A., Lorange, G. C., and Goldberg, H. (1957). A study of mitral stenosis by combined catheterization of the left and right sides of the heart. New England Fournal of Medicine, 256, I017.

Fox, I. J., Wakai, C. S., Connolly, D. C., and Wood, E. H. (1956). Left atrial and ventricular pressure pulses in mitral valvular disease. Proceedings of the Staff Meetings of the Mayo Clinic, 31, 126.

Gorlin, R., and Gorlin, S. G. (I95I). Hydraulic formula for calculation of the area of the stenotic mitral valve, other cardiac valves, and central circulatory shunts. I. American Heart fournal, 4I, I.

Werkö, L., Varnauskas, E., Eliasch, H., Lagerlöf, H., Senning, A., and Thomasson, B. (1953). Further evidence that the pulmonary capillary venous pressure pulse in man reflects cyclic pressure changes in the left atrium. Circulation Research, 1, 337.

Requests for reprints to Dr. K. E. Hammermeister, Cardiovascular Disease Service, Veterans Administration Hospital, 4435 Beacon Avenue South, Seattle, Washington 98108, U.S.A. 